\title{
Top ten biotechnologies for improving health in developing countries
}

\author{
Abdallah S. Daar ${ }^{1-4}$, Halla Thorsteinsdóttir1,2, Douglas K. Martinn,5,6, Alyna C. Smith 1,2, \\ Shauna Nast ${ }^{1,2}$ \& Peter A. Singer $2,4,7$
}

\begin{abstract}
Most research into genomics and other related biotechnologies is concerned with the priorities of industrialized nations, and yet a limited number of projects have shown that these technologies could help improve health in developing countries. To encourage the successful application of biotechnology to global health, we carried out a study in which we asked an international group of eminent scientists with expertise in global health issues to identify the top ten biotechnologies for improving health in developing countries. The results offer concrete guidance to those in a position to influence the direction of research and development, and challenge common assumptions about the relevance and affordability of biotechnology for developing countries.
\end{abstract}

\begin{abstract}
Most research in genomics and related biotechnologies (in the rest of this article, we refer to these simply as "biotechnologies") focuses on the needs of industrialized nations, a manifestation of the notorious ' $10 / 90$ gap' whereby $90 \%$ of health research dollars are spent on the health problems of $10 \%$ of the world's population $^{1}$. Although a few research teams scattered around the world are studying the application of new technologies to health problems of developing countries, their isolated efforts are unlikely to ensure equity of benefit. The World Health Organization (WHO) recently released a report titled "Genomics and World Health" that highlights the potential of genomics to improve global health. It recognizes that resources devoted to health research in developing countries are limited and that there is an urgent need to focus attention on the most promising technologies. The report recommended that WHO "should develop the capacity to evaluate advances in genomics, to anticipate their potential for research and clinical application ... and to assess their effectiveness and cost in comparison to current practice"2. An essential first step in addressing this recommendation is a technology foresight exercise to identify priority technologies. We have now completed a foresight study that identifies the ten most promising biotechnologies for improving health in developing countries in the next five to ten years. It is the first study to provide such information.
\end{abstract}

How the study was performed

We recruited a panel of 28 scientific experts from around the world (see Web Table A online) who are at the forefront of their fields; about half of them work in developing countries, and the remainder are either originally from developing countries or are well acquainted with public health problems of developing coun- tries. We chose to consult scientists rather than policy makers or other stakeholders because they are most familiar with current scientific research - a prerequisite for making the sort of judgments that this study requires. A conscious effort was made to balance the proportion of men and women and to represent scientists from various specialty areas and from a range of countries.

We began the study with an open-ended question: "What do you think are the major biotechnologies that can help improve health in developing countries in the next five to ten years?" Then, as the panelists responded, we asked them to identify the criteria driving their choices, which indicated that the panelists took the following factors into consideration when assessing the importance of the technologies.

Impact. How much difference will the technology make in improving health?

Appropriateness. Will it be affordable, robust and adjustable to health care settings in developing countries, and will it be socially, culturally and politically acceptable?

Burden. Will it address the most pressing health needs?

Feasibility. Can it realistically be developed and deployed in a time frame of 5-10 years?

Knowledge gap. Does the technology advance health by creating new knowledge?

Indirect benefits. Does it address issues such as environmental improvement and income generation that have indirect, positive effects on health?

We used the Delphi Method to achieve consensus ${ }^{3}$ (see Web Note A online). We worked with the panelists through several rounds by using combinations of personal interviews, e-mail messages, faxes and phone calls, and analyzed their input to produce the resulting priority list.

\footnotetext{
${ }^{1}$ Program in Applied Ethics and Biotechnology, ${ }^{2}$ Joint Centre for Bioethics, ${ }^{3}$ Departments of Public Health Sciences and Surgery, ${ }^{4}$ Pan American Health Organization/World Health Organization Collaborating Center for Bioethics, ${ }^{5}$ Collaborative Program in Bioethics, ${ }^{6}$ Department of Health Policy, Management and Evaluation and ${ }^{7}$ Department of Medicine, University of Toronto, 88 College Street, Toronto, Ontario M5G 1 L4, Canada. Correspondence should be addressed to P.A.S. (e-mail: peter.singer@utoronto.ca).
} 
The top ten

The results of the study are shown in Table 1. There was a high degree of consensus regarding the top three technologies: all but one panelist included at least one of these among their top three choices. Here we discuss the top three; all ten technologies are discussed in detail in a forthcoming report ${ }^{4}$.

The most highly rated category is "Modified molecular technologies for affordable, simple diagnosis of infectious diseases". Early, accurate diagnosis of infectious disease is important not only for prompt treatment, but also to limit the spread of disease and avoid the waste of resources on ineffective treatments. According to panelists, many diagnostic techniques currently in use in developing countries are cumbersome and unsuitable for use in low-resource settings. Molecular diagnostic technologies that are either already in use or are being tested in low-income regions include the polymerase chain reaction (PCR; ref. 5), monoclonal antibodies ${ }^{6}$ and recombinant antigens ${ }^{7}$. Modifications can make these technologies more suitable for the developing world; for example, a PCR-based HIV test that detects the presence of pro-viral DNA in infants has been simplified to use filter paper to process and store blood samples. The DNA can be amplified while the sample is bound to the filter paper, and samples stored this way are heat-stable and can be used for many months ${ }^{8,9}$. Simple hand-held test devices that rely on the binding specificity of monoclonal antibodies or recombinant antigens to diagnose infection may be easily adaptable to settings without running water, refrigeration or electricity 6,7 .

The second most highly rated category is "Recombinant technologies to develop vaccines against infectious diseases". Vaccines are a critical component of disease management in developing countries. Recombinant technologies are now at the forefront of efforts to produce new vaccines. For example, as part of the Malaria Vaccine Initiative, researchers have tested a subunit vaccine against malaria known as RTS,S/AS02. The phase 1 trial results were promising in adults in The Gambia ${ }^{10}$, and phase 2 trials are now underway in children in The Gambia and Mozambique ${ }^{11}$. Other examples mentioned by panelists include recombinant hepatitis $B$ vaccine ${ }^{12}$ and prime-boost vaccine strategies ${ }^{13}$. Some recombinant vaccines are already being manufactured in developing countries, sometimes at a fraction of the cost of the standard imported alternative ${ }^{12}$.

The third most highly rated category is "Technologies for more efficient drug and vaccine delivery systems". Most vaccines and many drugs are administered by injection, and yet tens of thousands of new cases of blood-borne diseases, such as HIV/AIDS and hepatitis $\mathrm{B}$, are caused each year by unsanitary injections ${ }^{14}$. The enormous expense of refrigeration (maintaining the required temperature can add up to $80 \%$ of the cost of vaccine delivery in developing countries) and the inconvenience of fre- quent dosing are two other drawbacks to current methods of vaccine and drug delivery ${ }^{15}$. Alternatives to injections, frequent dosing and refrigeration could increase safe access to drugs and vaccines, saving millions of lives ${ }^{16}$. Current alternatives include powdered vaccines, edible vaccines ${ }^{17}$ and controlled-release formulations that replace the need for multiple doses ${ }^{18}$.

\section{Assumptions about biotechnology and global health}

The results of our survey cast doubt on several common assumptions about the applicability of biotechnologies in developing countries. First, the assumption that biotechnology is irrelevant to the health needs of the world's poor is challenged by the panel's identification of tools that could help control illness in the developing world. Infectious disease can be controlled by molecular diagnostics (rated first) and recombinant vaccines (second). Recombinant therapeutic proteins (ninth) are also relevant to developing countries, where an epidemiological transition is in progress. Today, $60 \%$ of all deaths in developing countries are due to non-communicable diseases, and this figure is expected to reach $73 \%$ by 2020 (ref. 19). Malnutrition could be ameliorated using enriched genetically modified crops (eighth). Panelists also recognized the link between the empowerment of women and health, citing female-controlled protection against sexually transmitted disease (sixth), which can be addressed in part by genomics-based technologies ${ }^{20,21}$, as having potential to improve health in developing countries.

Second, our results discredit the assumption that biotechnology cannot contribute to the prevention of disease and the promotion of health. Vaccination is perhaps the best available form of prevention against infectious disease, and the new field of recombinant vaccines (rated second) is already making inroads where traditional vaccines have not been successful (for example, against malaria ${ }^{10}$ ). Alternatives to injections and vaccine refrigeration, covered under new systems of drug and vaccine delivery (third), can circumvent the need for refrigeration and reduce the number of new cases of blood-borne diseases caused each year by contaminated syringes. Technologies for environmental improvement (fourth), such as bioremediation, can help transform or sequester unhealthy pollutants in the soil or drinking water, improving public health.

Third, the results of this study suggest that biotechnology, especially molecular diagnositcs (rated first), can be made affordable for the developing world. Bioremediation (fourth) is usually less expensive than traditional methods of waste treatment or disposal ${ }^{22}$, and bioinformatics (seventh), the computer-based analysis of biological data (particularly gene sequences), is surprisingly affordable owing to free data, software and training available online $\mathrm{e}^{23,24}$. Enforcement of intellectual propertry rights will be crucial to the affordability of

\section{Table $1 \bullet$ The top ten biotechnologies with scores based on rankings of the expert panel}

\begin{tabular}{llr}
\hline Final ranking & Biotechnology & Final score \\
1 & Modified molecular technologies for affordable, simple diagnosis of infectious diseases & 288 \\
2 & Recombinant technologies to develop vaccines against infectious diseases & 262 \\
3 & Technologies for more efficient drug and vaccine delivery systems & 245 \\
4 & Technologies for environmental improvement (sanitation, clean water, bioremediation) & 193 \\
5 & Sequencing pathogen genomes to understand their biology and to identify new antimicrobials & 180 \\
6 & Female-controlled protection against sexually transmitted diseases, both with and without contraceptive effect & 171 \\
7 & Bioinformatics to identify drug targets and to examine pathogen-host interactions & 168 \\
8 & Genetically modified crops with increased nutrients to counter specific deficiencies & 159 \\
9 & Recombinant technology to make therapeutic products (for example, insulin, interferons) more affordable & 155 \\
10 & Combinatorial chemistry for drug discovery & 129
\end{tabular}


these technologies. Intellectual property is a complex subject plagued by confusion ${ }^{25}$. It is encouraging to note that, when the interests of developing countries are concerned, key stakeholders can be magnanimous ${ }^{26}$. The University of Ottawa and the University of Havana have decided to forego the royalties of a jointly developed pneumonia-meningitis vaccine in instances where it is used for strictly humanitarian purposes (R. Roy, personal communication). These sorts of arrangements are breaking new ground in intellectual property rights.

Though we emphasize here the usefulness of foresight and prioritization of promising biotechnologies, we do not wish to suggest that our top ten list comprises the only biotechnologies that have value for improving health in developing countries. Several technologies that scored high in the study but were not among the top ten include proteomics to target proteins and peptides that could be used in vaccines and therapeutic agents; DNA sequence analysis to discover population polymorphisms that may predispose to regionally specific diseases; gene-based drug screening technologies for local or traditional medicines aimed at providing affordable medicines while making the best use of local and natural resources such as indigenous plants and snake or insect venoms; and genetic modification of plants for production of common drugs (that is, using plants as bioreactors for drug production).

\section{An appropriate balance}

The intention of this study is to highlight the potential of biotechnologies for improving health in developing countries. This focus, however, should in no way diminish the importance of proven health strategies. Health education, for instance, is integral to the control of the AIDS pandemic, as is the provision and use of male condoms. Improvements in sanitation can markedly reduce the incidence of water-borne diseases, and basic nutritional education can help prevent nutrient deficiencies. These tools are available now, whereas the biotechnologies in our top ten list are in varying stages of development. Still, there is increasing evidence of the potential of these biotechnologies for improving the health of people in developing countries. A recombinant vaccine against HIV has successfully completed phase 2 clinical trials ${ }^{13}$. If this vaccine proves to be effective, affordable and culturally acceptable, it could be more successful at halting the spread of HIV than many current methods. Thanks to the sequencing of the genome of the malaria parasite Plasmodium falciparum, the drug fosmidomycin has moved in less than two years into clinical trials for the treatment of malaria. Fosmidomycin had already been approved for treating urinary infections; a systematic search of the parasite's genome revealed that it contains an enzyme known to be blocked by this drug 27,28 .

We ought, therefore, to strive to achieve an appropriate balance between such technologies and conventional strategies. This is not an easy task, but to ignore the potential of biotechnology is not the answer-not when there is strong evidence of its usefulness. Part of this balance will involve the appreciation that these technologies can be used to improve conventional public health strategies such as vaccines and sanitation.

\section{What next?}

This foresight study is a first step towards greater health equity through the application of biotechnology. A number of secondary steps flow immediately from these results. First, we encourage individual countries to assess the appropriateness of these technologies given their national contexts, and to focus on those technologies deemed to offer the greatest benefit. The process and criteria identified in this study can be used to guide these assessments.
We also encourage regional associations of developing countries to examine how they collectively can improve health in their regions by promoting the technologies suggested by our report. Our full report has been accepted for formal consideration by the Science and Technology Commission of the New Partnership for Africa's Development (NEPAD; J. Mugabe, personal communication). NEPAD represents a pledge among African leaders committed to the eradication of poverty and development of their region. It recognizes the importance of science and technology for sustainable development in Africa.

We urge pharmaceutical and biotechnology industry associations to work with their member companies to determine where the top ten biotechnologies sit in their product pipelines, to explore any impediments to their development, and to work with WHO and developing countries to address access issues for those technologies deemed suitable for diffusion. Such partnerships have been identified as a key strategy in the seminal report titled "Making New Technologies Work for Human Development" from the United Nations Development Programme ${ }^{29}$.

Our results can also be used to guide the policy formulation of major international donors and bilateral aid agencies such as the US National Institutes of Health, Rockefeller Foundation, Wellcome Trust, Gates Foundation and the proposed Global Health Research Fund ${ }^{30}$.

By providing concrete examples, this study focuses public attention on the benefits of genomics and other biotechnologies for developing countries and thereby sets the stage for more effective advocacy by WHO and others regarding harnessing biotechnology for developing world health.

The top ten list also focuses attention on those technologies that require further assessment. We encourage WHO to conduct a formal technology assessment of key examples from the top ten list of biotechnology platforms to determine their cost effectiveness. Moreover, we recommend that WHO repeat this global foresight exercise on a periodic basis (for example, every two to three years) to keep attention focused on the most promising technologies as the science develops. The process and criteria we have developed in this study would be useful for that purpose.

Foresight exercises such as ours have been found to increase communication, encourage the community to concentrate on the longer term, help foster better coordination among different stakeholders and develop a consensus on a shared future vision and commitment to specific goals ${ }^{31}$.

By focusing attention on the most promising biotechnologies, we have taken an important step beyond the WHO report on Genomics and World Health and down the path towards implementation.

URLs. Information on the Malaria Vaccine Initiative is available online at $\mathrm{http://www.malariavaccine.org}$, and the official website of NEPAD is located at http://www.nepad.com.

Note: Supplementary information is available on the Nature Genetics website.

\section{Acknowledgments}

We are grateful to the panel for providing the expertise to carry out this project; L.-C. Tsui, S. Scherer and C. Evans for help with our analysis of the technologies; A.J. Ivinson for editing this paper; and T. Pang, T. Evans, M. Fathalla, E. Dowdeswell and D. Yach for providing comments. Grant support was provided by the Program in Applied Ethics and Biotechnology (supported by the Ontario Research and Development Challenge Fund, GlaxoSmithKline, Merck and Co., Sun Life Financial, the University of Toronto, the Hospital for Sick Children, Sunnybrook and Women's College Health Sciences Centre and the University Health Network) and the 
Canadian Program on Genomics and Global Health (supported by Genome Canada). P.A.S. is supported by an investigator award from the Canadian Institutes of Health Research. The University of Toronto Joint Centre for Bioethics is a Pan American Health Organization/World Health Organization Collaborating Center for Bioethics.

\section{Received 8 July; accepted 4 September 2002}

1. Global Forum for Health Research. The $10 / 90$ Report on Health Research 2000 (Global Forum for Health Research, Geneva, 2000).

2. World Health Organization. Genomics and World Health (Report of the Advisory Committee on Health Research) (World Health Organization, Geneva, 2002).

3. Adler, M. \& Ziglio, E. Gazing into the Oracle: The Delphi Method and its Implications for Social Policy and Public Health (Jessica Kingsley, London, 1996).

4. Program in Applied Ethics and Biotechnology \& Canadian Program in Genomics and Global Health, University of Toronto Joint Centre for Bioethics. Top 10 Biotechnologies for Improving Health in Developing Countries (Univ. of Toronto Press, in press).

5. Harris, E. A Low Cost Approach to PCR: Appropriate Technology Transfer of Biomolecular Techniques (ed. Kadir, N.) (Oxford Univ. Press, New York, 1998).

6. Palmer, C.J. et al. Evaluation of the OptiMAL test for rapid diagnosis of Plasmodium vivax and Plasmodium falciparum malaria. J. Clin. Microbiol. 36, 203-206 (1998)

7. Aidoo, S. et al. Suitability of a rapid immunochromatographic test for detection of antibodies to human immunodeficiency virus in Ghana, West Africa. J. Clin. Microbiol. 39, 2572-2575 (2001).

8. Panteleeff, D.D. et al. Rapid method for screening dried blood samples on filter paper for human immunodeficiency virus type 1 DNA. J. Clin Microbiol. 37 350-353 (1999).

9. Beck, A.l. et al. Simple, sensitive, and specific detection of human immunodeficiency virus type 1 subtype B DNA in dried blood samples for diagnosis in infants in the field. J. Clin. Microbiol. 39, 29-33 (2001).

10. Bojang, K.A. et al. Efficacy of RTS,S/ASO2 malaria vaccine against Plasmodium falciparum infection in semi-immune adult men in The Gambia: a randomised trial. Lancet 358, 1927-1934 (2001).

11. Lee, E.A. et al. Induction of T helper type 1 and 2 responses to 19-kilodalton merozoite surface protein 1 in vaccinated healthy volunteers and adults naturally exposed to malaria. Infect. Immun. 70, 1417-1421 (2002).

12. Abraham, P. et al. Evaluation of a new recombinant DNA hepatitis $B$ vaccine (Shanvac-B). Vaccine 17, 1125-1129 (1999)

13. Belshe, R.B. et al. Safety and immunogenicity of a canarypox-vectored human immunodeficiency virus Type 1 vaccine with or without gp120: a phase 2 study in higher- and lower-risk volunteers. J. Infect. Dis. 183, 1343-1352 (2001).

14. Kane, A., Lloyd, J., Zaffran, M., Simonsen, L. \& Kane, M. Transmission of hepatitis $B$, hepatitis $C$ and human immunodeficiency viruses through unsafe injections in the developing world: model-based regional estimates. Bull. World Health Organ. 77, 801-807 (1999).

15. World Health Organization. Fact Sheet 169: Global Alliance for Vaccines and Immunization (World Health Organization, Geneva, 2001; http://www.who.int/ inf-fs/en/fact169.html).

16. Jha, P. et al. Improving the health of the global poor. Science 295, 2036-2039 (2002).

17. Langridge, W.H.R. Edible vaccines. Sci. Am. 283, 66-71 (2000).

18. Jodar, L. et al. Ensuring vaccine safety in immunization programmes-a WHO perspective. Vaccine 19, 1594-1905 (2001).

19. World Health Organization. Fact Sheet 106: Noncommunicable Diseases: WHO Experts Warn Against Inadequate Prevention, Particularly in Developing Countries (World Health Organization, Geneva, 1996; http://www.who.int/inf-fs/ en/fact106.html)

20. Boyd, M.R. et al. Discovery of cyanovirin-N, a novel human immunodeficiency virus-inactivating protein that binds viral surface envelope glycoprotein gp120: potential applications to microbicide development. Antimicrob. Agents Chemother. 41, 1521-1530 (1997).

21. Kelley, B.S., Chang, L.C. \& Bewley, C.A. Engineering an obligate domain-swapped dimer of cyanovirin-N with enhanced anti-HIV activity. J. Am. Chem. Soc. 124, 3210-3211 (2002).

22. United States Environmental Protection Agency. Citizen's Guide to Bioremediation (U.S. Environmental Protection Agency, Washington, DC, 2001).

23. Butler, D. Bioinformatics to be nurtured online. Nature 411,513 (2001).

24. Benson, D.A. et al. GenBank. Nucleic Acids Res. 28, 15-18 (2001).

25. Gold, R.E., Castle, D., Cloutier, L.M., Daar, A.S. \& Smith, P.J. Needed: models of biotechnology intellectual property. Trends Biotech. 20, 327-329 (2002).

26. Normile, D. Monsanto donates its share of Golden Rice. Science 289, 843-845 (2000).

27. Jomaa, H. et al. Inhibitors of the nonmevalonate pathway of isoprenoid biosynthesis as antimalarial drugs. Science 285, 1573-1576 (1999).

28. Wiesner, J. et al. Structure-activity relationships of novel anti-malarial agents. Part 3: N-(4-Acylamino-3-benzoylphenyl)-4-propoxycinnamic acid amides. Bioorg. Med. Chem. Lett. 25, 543-545 (2002).

29. United Nations Development Programme. Human Development Report 2001: Making New Technologies Work for Human Development (Oxford Univ. Press, New York, 2001)

30. World Health Organization. Macroeconomics and Health: Investing in Health for Economic Development (Report of the Commission on Macroeconomics and Health) (World Health Organization, Geneva, 2001).

31. Martin, B.R. Foresight in science and technology. Technol. Anal. Strateg. Manage. 7, 139-168 (1995). 